\title{
Considerations for the Medical Management of the Circus Performance Artist and Acrobat
}

\author{
John Faltus, DPT, MS, SCS, ATC, $\operatorname{CSCS}^{1}$ a , Veronique Richard, PhD ${ }^{2}$ \\ ${ }^{1}$ KBR, Fort Bragg, NC, USA, ${ }^{2}$ National Circus School, Montreal, Canada \\ Keywords: performance arts, acrobat, circus medicine \\ https://doi.org/10.26603/001c.31645
}

\section{International Journal of Sports Physical Therapy}

Vol. 17, Issue 2, 2022

\begin{abstract}
Medical management of the circus performer encompasses a wide variety of multicultural, transdisciplinary and multifaceted decision-making considerations. There is a paucity of research evidence investigating both the unique diversity of skill sets and cultural considerations in addition to injury patterns of performers within the circus environment. Since a previously established framework for supporting the health and well-being of the circus performer across various aspects of medical management does not exist in the literature, most recommendations in this regard must come from practical experience working with this highly specialized performance athlete population. The purpose of this clinical commentary is to provide the reader with a greater understanding of the unique challenges associated with the medical management of performance artists and acrobats as well as recommendations for developing an integrated approach for mitigating injury risk within a highly specialized, diverse athlete population.
\end{abstract}

\section{Level of Evidence}

5

\section{INTRODUCTION}

Circus performance encompasses a wide variety of disciplines, ranging from acrobats, artistic characters, former Olympic-level and professional athletes, and musicians, where unique skill sets are creatively integrated with artistic components and athletic ability to create show performance. ${ }^{1-3}$ Further, within performance acrobats, the individual performers are highly specialized with backgrounds which include ballet, dance, gymnastics, and acrobatic sport. As a result of these specializations and performance demands, the medical management of these performers requires an understanding of the complex interaction of both discipline specific considerations and performance act requirements. Despite the complexities and uniqueness of the circus performance environment, an established and evidence-supported framework for managing the health and well-being of this performance population does not exist. Therefore, recommendations and suggestions to the field in regards to implementation of a multi-faceted medical management program are primarily anecdotal and based upon practical experience. This commentary will focus on providing the reader with a greater understanding of the unique challenges associated with the medical management of performance artists and acrobats as well as recommendations for developing an integrated approach for mitigating injury risk within a highly specialized athlete population that is diverse in many aspects.

\section{NEEDS ANALYSIS}

Given the diversity of performer backgrounds within the circus environment, performance demands and functional capacities of each performer and their performance acts must be studied closely. Nomenclature specific to performance arts includes "artist" in place of "athlete”, however performance artists include both acrobatic and non-acrobatic performers. For the sake of clarity, performance artists, or non-acrobatic performers, and performance acrobats (Figure 1) must be distinguished separately within the framework of this clinical commentary. Further, these disciplines have been previously characterized as either acro- 
bats, non-acrobats or musicians. ${ }^{1}$

In order to further distinguish between types of performers, a classification system of either sudden load performers, non-sudden load performers and musicians has also been used. ${ }^{4}$ Sudden load performers would be those who incur a sudden, substantial increase in physical compression or distraction loads as previously defined by Orlando et al. ${ }^{4}$ Skill sets such as diving, gymnastics, trampoline, partner lifting or catching, hand balancing, contortion (Figure 2), ballistic style dancing and aerial movements would be categorized as sudden load. 1,4

Non-sudden load performers may include jugglers (Figure 3), artistic image dancers and clowns, however features within the skill sets of these performers may also be dynamic and sudden load in nature, indicating a diversity in skill sets across all performer disciplines. ${ }^{1}$

Beyond the primary act requirements specific to a performer's discipline, a show performance also includes cue tracks, or minor performances such as brief dancing, character or image expression sequences specific to creating an artistic effect on stage for a particular act. While the cues required for each show may vary and generally have performer rotations, they do require specific movement demands which may or may not be similar to those of the performer's specific act while contributing to overall performance workload. ${ }^{3}$ For example, a dancing animal cue which requires patterns of repeated lateral movement or sustained trunk flexion contrasts with the demands of overhead traction and torsion placed on the shoulder of a high bar acrobat who may be required to perform both in show performance. The ability to perform at a high-level within their discipline-specific act while also mastering artistically influenced movement patterns required of their cue track demands adaptability, continued refinement and versatility within the performer's skill set. It is also important to recognize that no two acrobats have the same load demands within a performance act, despite similar backgrounds and disciplines, as implementation of their skill set within an act is greatly influenced by the timing of choreographic sequences, which are integrated for the sake of artistic expression.

\section{INJURY RISK MITIGATION CHALLENGES}

While one of the earliest studies on injuries in the circus performer population found injury rate to be lower in comparison to similar sport disciplines within the National Collegiate Athletic Association, ${ }^{1}$ research on this unique and diversified demographic has evolved. ${ }^{2,3}$ With show opportunities expanding and performers becoming more specialized, injury risk is more apparent and worthy of further discussion and investigation. ${ }^{2,3}$ Performance in circus requires high workloads through repetitive training and frequent show performance exposures which creates injury risk comparable to that in sports, with 7.3 to 9.7 injuries per 1000 artist performances. ${ }^{1-3,5}$ These injury rates in circus are comparable to those found in a study of practice injuries in NCAA sports, in particular Fall season sports which demonstrated injury rates of 7.4 injuries per 1000 exposures with the highest injury risk sport being women's soccer at 9.3 injuries per 1000 exposures. ${ }^{5}$ The diversity of disciplines

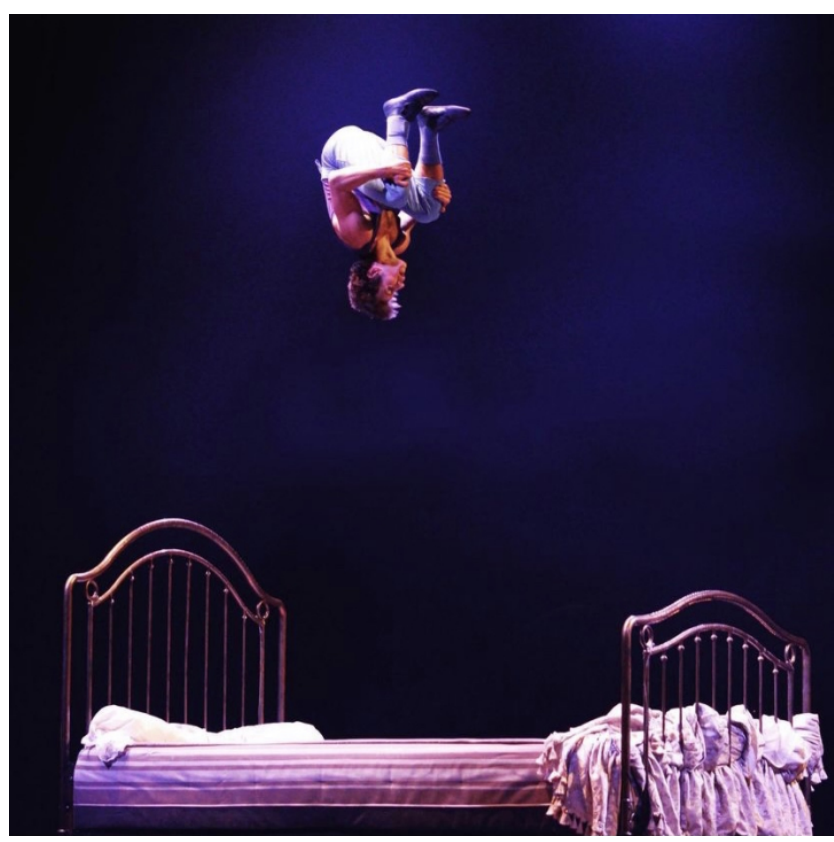

Figure 1. A performance acrobat defies gravity on the trampobed

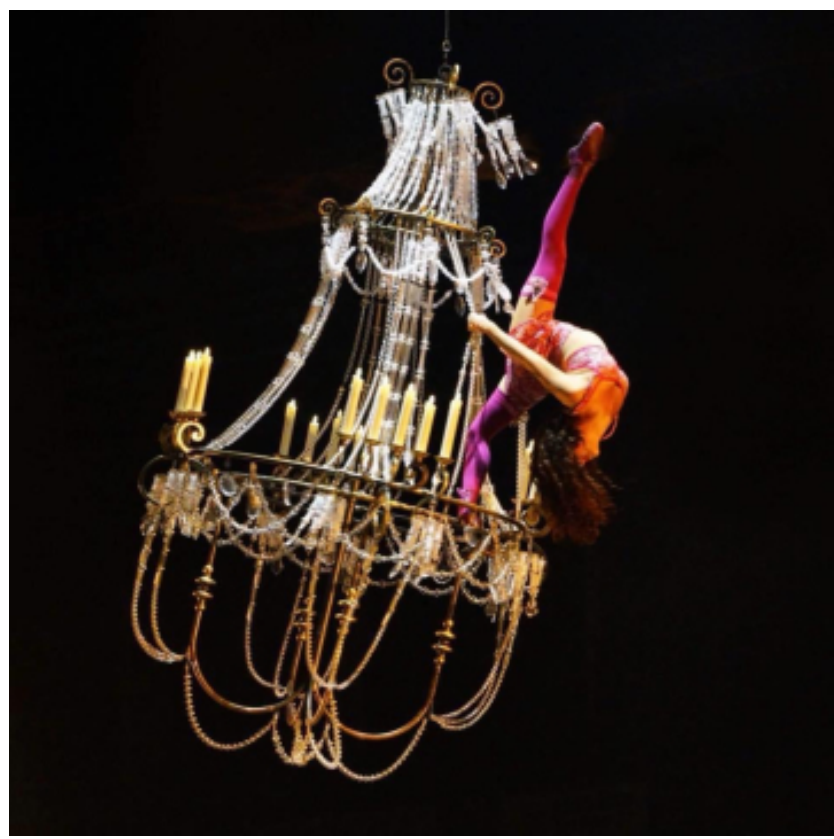

Figure 2. A contortion artist challenges the limits of flexibility on the aerial chandelier

within both acrobatic and non-acrobatic performer profiles further influences a unique variety of injury conditions and presentations which require creativity while progressing an injured performer back to show performance. ${ }^{1-4}$ These high workloads within circus performance require frequent, repetitive movements and dynamically loaded patterns through extreme ranges of motion which contribute significantly to time-loss from training and performance due to a variety of acute, traumatic, and chronic injuries. ${ }^{1,2,6}$

Show exposures may consist of 8-10 shows per week over 
the course of 5-6 consecutive days, not including “off-days" that may require international travel by bus, air or train to the next tour city which can negatively influence sleep quality, recovery, performance and injury risk as shown in a variety of athletic populations. ${ }^{3,7-10}$ Fluctuating show performance times across various time zones within all seasons are variables that can negatively influence performance and recovery. Further, due to the nature of training sessions and show performances being held indoors across a variety of global venues at various times of year, lack of sun exposure and vitamin D deficits, especially during the winter and early spring seasons, must be considered as potential contributors to injury. ${ }^{10}$ Given the complexity of show performance and lifestyle demands, injury risk within the circus performer population is a constantly evolving variable that needs to be monitored and managed, especially within a highly specialized show performance environment where "backup performers" and shared allocation of show performance "minutes", in load management vernacular, are not always available and possible.

Another unique challenge of mitigating injury risk as it relates to the volume and frequency of circus performance training and show exposure is the lack of a true "offseason" period. Unlike professional sport where offseason training commences following the conclusion of the competitive season, circus performances continue year-round with variable, brief show performance "breaks" ranging from a few weeks or longer and occurring between tour legs, which typically consist of a 9-12-week period of continuous touring and an intensive show performance schedule. Further, given the unique nature of what performers are required to do on stage as well as the specific equipment and technical aspects required within their acts, access to these apparatuses during these performance breaks is not always ensured which could contribute to injury risk when resuming training and show performance if there is a period of act specific "de-loading". These exposures to the potential risk factors of 1) fluctuations in act-specific training 2) decreased loading patterns both during and after sporadic tour and show performance breaks and 3) high volume training and show performance workloads over the course of a prolonged period of time can significantly, through workload spikes following periods of decreased training, contribute to injury risk in the circus performer as demonstrated in other athletic populations. ${ }^{11-15}$ Further, depending on performance group and entertainment company size, the exposure risk may be heightened when back-up performers are not available across the show and/or for a particular act which would then require the highly specialized performers to perform in their assigned role during each show, essentially compromising periods of relative rest where said performers would still perform but in a reduced capacity on a show-by-show rotation basis. In the presence of larger casts with built-in act and show performance rotations, the ability to implement these relative rest periods may potentially become more practical. However, even in this ideal situation, injuries across the show can alter line-ups and result in modifications to even the best planned rest-rotation schedule.

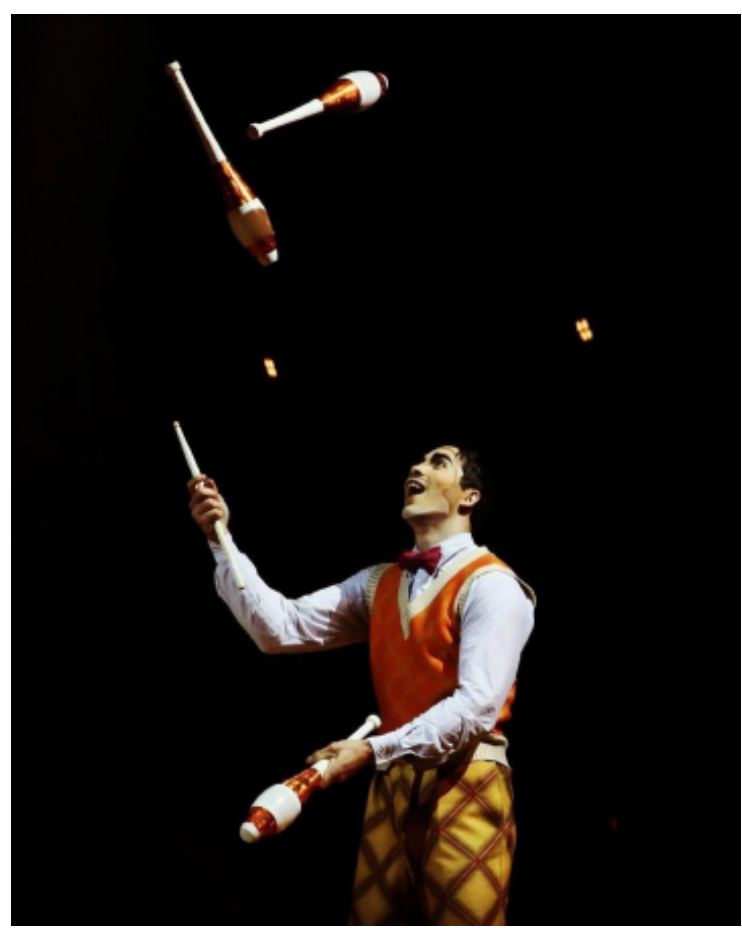

Figure 3. A juggler skillfully combines finesse and showmanship

\section{CULTURAL CONSIDERATIONS}

Given the diversity of cultures represented within circus performer disciplines, it is important for the practitioner to be sensitive to varying levels of understanding and interpretation of injury by the performer. In some cases, pain may be perceived as an acceptable or expected aspect of performing, a "badge of honor" in some respects, that is not deemed as threatening in the current moment. Some performers would attempt to perform through pain and disregard medical opinion rather than feel as if they "let down" the fellow members of their performance team or act. However, it is in these situations where the intersection of medical recommendations and emotional intelligence of the practitioner must be most robust and steadfast as prolonged injury conditions without proper medical evaluation can result in extensive time loss due to injury and potentially career threatening injuries. Guided by a collaborative decision-making approach which includes the coach and associate stakeholder who can assist with language translation as needed, education on the risk of career endangerment and harm potentially which can be caused to fellow performers within an act when a performer is not at full health should be broached in these discussions to ensure better understanding. Despite the existence of self-induced pressure to perform even with a significant musculoskeletal injury, show tracks and cues can be modified without creating increased risk to injured performers but still allowing them to perform in some capacity in show performances. These aspects of determining show performance status further illustrate the need for a collaborative, understanding and openly communicative decision-making structure within the circus environment. 
As it relates to consultant-provided medical care, standards, systems and injury management recommendations vary significantly on a global scale. The interpretation of and recommended treatment interventions for a variety of injury conditions can vary greatly, with the heightened risk associated with circus acrobats in particular heavily influencing what may be perceived as more conservative timelines and restrictive recommendations.

Further influencing dynamics of the performer-practitioner healthcare relationship are the cultural expectations for what information relevant to their injury is expected, what is considered important in terms of how an injury is described and managed, and how involved the individual desires to be in the decision-making process while engaging with their practitioner. In some cultures, "less is more" in that minimal information is preferred as it relates to best understanding an injury condition and pathology. ${ }^{16,17}$ Misunderstandings or lack of awareness when educating and engaging the performer in their own healthcare can occur if the practitioner is not sensitive to cultural differences and language discrepancies, negatively influencing injury management outcomes and producing intercultural communication conflicts. ${ }^{17-20}$ Acknowledging and understanding potential differences in beliefs regarding health, injury or illness across a variety of ethnic groups is likely to reduce gaps in practitioner-performer communication and ensure a more positive outcome. ${ }^{19,20}$ For example, navigating the medical referral process for a Russian performer primarily speaking Russian and minimal English who sustained a shoulder dislocation injury on tour in Japan and is being seen by a local doctor who speaks neither English nor Russian will require linguistic diplomacy, planning, and levelheadedness to ensure a desirable outcome. Certainly, the utilization of an established global medical referral network or telehealth services could meet the needs of the injured performer and provide diagnostic clarification in situations where language discrepancy or limited medical specialty services exist.

\section{PSYCHOLOGICAL CONSIDERATIONS}

Due to the unique interaction of both athletic ability and artistic expression required of the circus performance artist and acrobat, the emotional, social, and cognitive loads experienced by these performers needs to be considered.

Injuries as well as other critical circus situations such as on-stage accidents, show cancellations, and relationship issues can elicit a myriad of emotional responses which impact the health of artists. Furthermore, contrary to most sports, circus requires a deep level of emotional embodiment. ${ }^{21}$ Hence, providing an emotionally safe environment is paramount in supporting the creative growth and performance of the performers. ${ }^{22}$ In this regard, researchers have found that emotional regulation is integral to performing artist success and that it can reduce the occurrence of accidents and near misses in contemporary circus arts. ${ }^{22,23}$ It is thus essential for practitioners to address the emotional experiences of a significant event to help artists recognize, understand, label, express, and regulate emotional content attached to the experience. Active listening where the practitioner expresses curiosity by asking questions rather than making judgements can be a powerful tool. Processing emotional experiences, both verbally and through movement, is a crucial step towards enhancing psychological functioning and wellbeing in performing artists. In fact, the absence of emotional regulation support can result in long lasting emotional consequences thereby affecting the likelihood of developing a variety of health problems. ${ }^{24}$

Many circus acts are performed within a team environment and, therefore, optimal performance is influenced by the development of shared coordination and cohesion among teammates. ${ }^{25}$ Because safety at times depends on coordinated precision among artists as well as technicians, band leaders, and stage managers, it is thus important to stay attuned as practitioners to the quality of the social interactions experienced by artists. Lack of team cohesion and coordination increases social anxiety levels and mistrust within a team and could, for instance, reduce work enjoyment, slow down the reintegration process after an injury, and impede performance. ${ }^{22,26}$ In addition, there may be limited opportunities for socialization outside of the touring show environment, increasing the negative impact of poor social connections on artists' overall wellbeing. Establishing team processes and support systems to foster trust, respect, and effective communication amongst artists is key. ${ }^{22,27}$

Improved awareness and understanding of socio-cognitive skills can favor best practice within a circus medical management program. For instance, it has been shown that artists with low self-efficacy are twice as likely to get injured compared to their higher self-efficacy counterparts. Specifically, a low level of either success or personal accomplishment may be predictive of injury. ${ }^{28}$ Lower self-efficacy is also associated with elevated anxiety and fear of failure and injury which has direct impact on motor performance because of its influence on attentional control. ${ }^{22}$ Specifically, elevated anxiety can make artists more inclined to reinvest their knowledge and overthink. This "paralysis by analysis" phenomenon disrupts movement automaticity resulting in a robotic performance and lower overall performance quality. In some cases, this disruption in automatic cognitive patterns can lead to Lost Movement Syndrome (LMS), a psychological condition in which athletes find themselves unable to perform a skill that was previously automatic. Research shows that this condition can negatively influence career potential. ${ }^{29}$ It is important to note that some individuals are more prone to performance deterioration under elevated anxiety. For instance, perfectionism, ruminating thoughts, and knowledge reinvestment have been identified as potential psychological mechanisms influencing motor performance decline. Hence, mental skills training can be highly effective within the circus community to enhance cognitive functioning. $22,26,30$

Contrary to sports where success is determined by an end-score regardless of spectator involvement, performing arts strive to immerse the audience into the performance of the artists. Hence, stage performance requires unique mental capacity to produce both captivating motor tricks and artistic display to evoke a crowd response which greatly influences how success may or may not be defined. Beyond what would be required on stage, the 'circus life' demands described through this commentary (e.g., frequently chang- 
ing country, venue, and lodging environments) may naturally impose psychological demands and challenges on the performer which can influence fatigue, emotional wellbeing, on-stage performance, and injury risk and/or presentation. ${ }^{28}$

\section{RECOMMENDATIONS}

Given the diversity of skill sets and backgrounds possessed by the circus performer, the clinical examination, treatment, and return to performance decision-making processes must consider both the unique demands required of a highly specialized performance cohort in addition to the wide-ranging influence and vested interest of numerous stakeholders who are involved in ensuring an optimal performance environment for these performers. Successful medical management of the circus performer should thus account for a wide variety of considerations that influence injury mitigation, health and performance. ${ }^{3}$

\section{COMMUNICATION AND COLLABORATION}

Bolling et al. have thoroughly described and outlined the complex and dynamic interactions between circus stakeholders, ranging from technicians and wardrobe personnel to stage managers and the artistic team, as they relate to performer healthcare management. ${ }^{3}$ Namely, they highlighted that effective communication and collaboration strategies facilitate healthcare management when multiple, diverse professionals are involved. In the circus environment, it is advised to adopt a flexible mindset that is sensitive to the "when and where" aspects of effective communication. This requires an open-minded, ego-quiet, and understanding attitude. For example, as it would relate to understanding the perspective of a member of the wardrobe department, it may not be so relevant as to why a performer's shoulder range of motion would need to be limited due to an acute acromioclavicular joint sprain but, rather, what modifications would be needed within the costume to protect this joint from possible compromise or what permissible color and style of supportive taping that can be applied by the medical department while still maintaining a certain required aesthetic on stage. On the contrary, it would be crucial for the act coach to know what range of motion limitations are present in order to possibly modify act sequences which still allows the performer to safely participate and not at the cost of aggravating the current condition further.

Internally, daily meetings and correspondence via medical reports with artistic directors, stage managers, and coaches provides a medium for open dialogue and feedback regarding medical management strategies on a case-bycase basis. Availability and open-mindedness are critical in not only strengthening communication with both the performer and stakeholders but allows the medical provider to seek out a greater understanding of the nuances and unique act specific demands relative to that performer's particular role within the show performance. Robust communication practices between medical providers and coaching staff are likely to yield more favorable outcomes relating to injury and availability. 31

Developing a global medical network external to the show can be a tremendous resource for accessing both local and international medical providers and should include a variety of practitioners, including, but not limited to, general medical doctors, orthopedic doctors, sports psychologists, nutritionists, chiropractors, and massage therapists. Given the unique demand of frequently moving show tours combined with the cultural aspects of circus performer medical management, it is important to integrate external medical providers who have either previously been exposed to managing circus performer injuries in the past or regularly consult with athletes in high performance sport to best comprehend the very unique set of skills and considerations when medical recommendations are provided to and for an injured performer. Virtual or telemedicine and translator services associated with these consultations with preferred doctors and specialized health providers present an opportunity to still access reliable and trusted medical care remotely when language differences and medical practice standards vary greatly on a global scale. Providing the consulting doctors with information in advance that is pertinent to the unique act demands and specialization of the performer, specifically through video of a particular act or injury episode, as well as relevant considerations for returning the performer back to full show performance in regards to cue track, act sequences and co-performer interactions, is recommended.

\section{TRANSDISCIPLINARY APPROACH}

The communication and collaboration styles described above are in line with a transdisciplinary approach. According to Karol et al., ${ }^{32}$ within this approach professionals that are part of the rehabilitation team defined patient needs collaboratively using their unique knowledge and experiences. It moves away from the "quantitative, typically reductionist and mono-disciplinary approaches to physiology, motor-learning, biomechanics, and psychology." 33 In sport, transdisciplinary rehabilitation teams are usually composed of physical, psychological, technical, and managing professionals plus the athlete. ${ }^{34}$ Including the athlete across the entire rehabilitation process is at the core of this athlete-centric approach as it was shown to increase athletes' empowerment, engagement, and motivation in addition to decreasing burnout for sport medicine practitioners. ${ }^{35}$ Further, environments which foster collaboration and trustworthiness amongst staff while providing athletes with a positive vision, clear communication and continued support throughout training programs resulted in more favorable outcomes in regards to injury reduction and increased game availability. ${ }^{36}$

At a 'micro-level', adopting a transdisciplinary approach can translate into rehabilitation programs which integrate neurophysiologic and neurocognitive aspects of injury. ${ }^{37,38}$ In circus, such programs would entail educating the performer on information processing, perceptions, sensations, and emotions experienced during the task. Then, the performer and medical provider, with the collaboration of the strength \& conditioning coach, mental performance specialist and artistic coach, can generate ideas to design in- 
novative and engaging rehabilitation exercises that are representative of the physical, cognitive, affective, and social aspects of a particular skill or performance specialty. The rehabilitation program is thus more holistic in nature and not solely focused on the physical aspects of the healing process. Although promising, this approach deserves further empirical research to clearly highlight the depth of benefits.

At the macro-level, transdisciplinary processes consider the complex interactions of socio-ecological systems and could better support a health enhancement program. Bolling et al identified three injury prevention strategies in the realm of circus: safety, load management and preparation. ${ }^{3}$ While the strategies described were mainly physical (e.g., performing exercises) or organizational (e.g., adapting the schedule), expanding the load management and preparation strategies to include the cultural, social, cognitive, and affective aspects is also recommended.

Given what may be a condensed schedule during show weeks, training time for individual and group acts may either be limited or not readily available depending on the tour plan dynamics, facility lay-outs and technical inspection requirements which ensure proper safety of performance apparatuses. Therefore, traditional periodization and load management methods used in sports may not necessarily be applicable for the circus environment. It is important to consider that load demands in the circus population are not uniform, vary across disciplines and require varying management strategies across these disciplines. For example, a contortionist troupe may benefit from repeated, high volume static load exposures in both training and show performance to maintain soft tissue pliability whereas trampoline acrobats would require a more closely monitored and orchestrated training plan to ensure that training loads do not compromise their ability to recover and perform in shows given the ballistic, repetitive nature of their performance act.

In addition to the specificity of the task to be performed, contextual situations can also greatly impact the perceived load of a performance schedule. For instance, the cognitive load of learning new cues in the show can impact the level of fatigue of the artist, even if the cues are not physically demanding. Similarly, trying a high-risk trick for the first time might increase anxiety level, emptying the artist's energy 'tank' faster. Finally, conflicts arising between team members or partners can be socially heavy to manage, which are also influenced by cultural background. Paying attention to the cognitive, affective, and social load/demands can help practitioners better design holistic preventive plans. By working in harmony with other specialists whom are both internal and external to the show, increased psychological support can be planned when the performance schedule involves high cognitive demands. Relaxation, meditation, or mindfulness sessions can be integrated to the daily schedule to help alleviate anxiety in addition to implementing a psychologically safe space to help artists communicate among themselves to avoid or manage social tension. According to the socio-ecological model of resilience 39 "environments that provide resources to develop or maintain optimal psychological, social, and physical wellbeing facilitate the capacity of individuals to withstand, overcome, and adapt to adversity". ${ }^{40}$ This type of community-based approach is relevant to professional circus contexts as it ensures the development of both internal and external resources, so the individual feels fully supported. 41

The development of resources can also optimize preparation prior to show. Warmups which encourage artists to activate both their body and minds while synchronizing familiar performance sequences with each other is thus recommended. Although research is scarce about the impact of holistic warmups, ${ }^{42}$ the authors recommend providing performers with a wide variety of tools for them to build an individualized preparation for performance. Educational resources by way of infographics translated to a variety of languages spoken on tour and posted on-site, guest speakers, periodic group or "question and answer" sessions, and daily interactions during both ongoing injury management sessions and treatments can be provided to best inform artists. This knowledge can then influence prudent decision making on the part of the performer as it relates to adequate preparation as well as other important habits which influence sleep, recovery, emotional well-being, nutrition, and hydration while on tour to mitigate injury risk. ${ }^{9}$

Because injury prevention is considered to be a dynamic and complex system, one must consider how elements of this system interact and change over time. ${ }^{3}$ Hence, the use of a subjective wellness and preparation questionnaire, such as a multi-item Likert scale which provides ratings for general fatigue, muscle soreness, emotional states, coping resources, and sleep quality, can be used to assess perceived recovery from previous training as well as readiness for same-day participation. ${ }^{43-45}$ Training plans can be effectively developed and planned according to show volume and context, act and cue rotations, and "stage" or training area availability each week, rather than over the course of an entire tour plan due to the spontaneous and ever-changing nature of the circus environment. Input provided from a multidisciplinary team of stage managers, artistic coaches, performer-coaches, show directors, technicians and medical providers can promote a transdisciplinary approach in managing training and show load exposures not just within individual performance acts but across the performance cast as a whole.

Future research should focus specifically on the individual cohorts represented within this unique performance population to further investigate the needs analysis and mental performance demands as well as injury patterns which may vary between acrobats, artistic characters, and musicians. Cohort studies focused on these varying performance groups will allow for injury surveillance and treatment protocols to become more specialized and ideally both enhance performance and reduce injury risk.

\section{CONCLUSION}

Medical management of the circus performer requires a transdisciplinary, multicultural, and multifaceted approach. The skill sets and performance requirements of circus performers are diverse and highly specialized with a wide variety of injury conditions which present. Collaboration and establishing clear lines of communication between 
and with non-medical essential stakeholders, such as stage managers, artistic directors, coaches, and technicians, is integral to managing the many aspects of circus life which influence the healthcare and wellbeing of the performer. Effective medical management should consider social and cultural perspectives as it relates to interpretation and coping strategies related to injury as well as the unique psychological and physical load demands placed upon the circus performer. An established global medical network and educational initiatives can be instrumental in guiding the understanding and managing of injury as well as fostering a pro-active approach for achieving optimal performance, health, and well-being of the circus performer.

\section{ACKNOWLEDGMENTS}

The authors would like to acknowledge and thank Brett Whyborn and Evert Verhagen for their contributions during the manuscript development process.

Submitted: April 10, 2021 CST, Accepted: November 09, 2021

CST 


\section{REFERENCES}

1. Shrier I, Meeuwisse WH, Matheson GO, et al. Injury patterns and injury rates in the Circus arts: An analysis of 5 years of data from Cirque du Soleil. Am J Sports Med. 2009;37(6):1143-1149. doi:10.1177/03635 $\underline{46508331138}$

2. Wolfenden H, Angioi M. Musculoskeletal injury profile of Circus artists: A systematic review of the literature. Med Probl Perform Art. 2017;32(1):51-59. d oi:10.21091/mppa.2017.1008

3. Bolling C, Mellette J, Pasman HR, van Mechelen W, Verhagen E. From the safety net to the injury prevention web: Applying systems thinking to unravel injury prevention challenges and opportunities in Cirque du Soleil. BMJ Open Sport Exerc Med. 2019;5(1):e000492. doi:10.1136/bmjsem-2 018-000492

4. Orlando C, Levitan EB, Mittleman MA, Steele RJ, Shrier I. The effect of rest days on injury rates. Scand J Med Sci Sports. 2011;21(6):e64-e71. doi:10.1111/j.160 0-0838.2010.01152.x

5. Agel J, Schisel J. Practice injury rates in collegiate sports. Clin J Sports Med. 2013;23(1):33-38. doi:10.109 7/jsm.0b013e3182717983

6. Munro D. Injury patterns and rates amongst students at the national institute of circus arts: An observational study. Med Probl Perform Art. 2014;29(4):235-240. doi:10.21091/mppa.2014.4046

7. Forbes-Robertson S, Dudley E, Vadgama P, Cook C, Drawer S, Kilduff L. Circadian disruption and remedial interventions: Effects and interventions for jet lag for athletic peak performance. Sports Med. 2012;42(3):185-208. doi:10.2165/11596850-00000000 0-00000

8. Huyghe T, Scanlan A, Dalbo V, Calleja-González J. The negative influence of air travel on health and performance in the national basketball association: A narrative review. Sports. 2018;6(3):89. doi:10.3390/sp orts6030089

9. Walsh N, Halson S, C S, et al. Sleep and the athlete: Narrative review and 2021 expert consensus recommendations. Br J Sports Med. 2020;0:1-13.

10. Gao B, Dwivedi S, Milewski M, et al. Chronic lack of sleep is associated with increased sports injury in adolescents: A systematic review and meta-analysis. Orthop J Sports Med. 2019;7(Suppl.

S3):232596711900132.
11. Gabbett TJ. The training-injury prevention paradox: Should athletes be training smarterandharder? Br J Sports Med. 2016;50(5):273-280. doi:10.1136/bjsports-2015-09578 $\underline{8}$

12. Cross MJ, Williams S, Trewartha G, Kemp SPT, Stokes KA. The influence of in-season training loads on injury risk in professional rugby union. Int J Sports Physiol Perform. 2016;11(3):350-355. doi:10.1123/ijsp p.2015-0187

13. Dennis R, Farhart R, Goumas C, Orchard J. Bowling workload and the risk of injury in elite cricket fast bowlers. J Sci Med Sport. 2003;6(3):359-367. doi:10.1016/s1440-2440(03)8003 $\underline{1-2}$

14. Stares J, Dawson B, Peeling P, et al. Identifying high risk loading conditions for in-season injury in elite Australian football players. J Sci Med Sport. 2018;21(1):46-51. doi:10.1016/j.jsams.2017.05.012

15. Colby MJ, Dawson B, Heasman J, et al. Preseason workload volume and high-risk periods for noncontact injury across multiple Australian Football. J Strength Cond Res. 2017;31(7):1821-1829. ㅁ oi:10.1519/jsc.0000000000001669

16. Blackhall LJ, Murphy S, Frank G, et al. Ethnicity and attitudes toward patient autonomy. JAMA. 1995;274(10):820-825. doi:10.1001/jama.1995.035301 $\underline{00060035}$

17. Seijo R, Gomez H, Freidenberg J. Language as a communication barrier for Hispanic patients. Hispanic J Behav Sci. 1991;13(4):363-373. doi:10.1177/ 07399863910134001

18. Rivadeneyra R, Elderkin-Thompson V, CohenSilver R, Waitzkin H. Patient centeredness in medical encounters requiring an interpreter. $\mathrm{Am} \mathrm{J} \mathrm{Med.}$ 2000;108(6):470-474. doi:10.1016/s0002-9343(99)004 $\underline{45-3}$

19. Cass A, Lowell A, Christie M, et al. Sharing the true stories: Improving communication between Aboriginal patients and healthcare workers. Med J Australia. 2002;176(10):466-470. doi:10.5694/i.1326-5 377.2002.tb04517.x

20. Schouten BC, Meeuwesen L. Cultural differences in medical communication: A review of the literature. Patient Educ Couns. 2006;64(1-3):21-34. doi:10.1016/ j.pec.2005.11.014 
21. Ross A, Shapiro J. Under the big top: An exploratory analysis of psychological factors influencing circus performers. Perform Enhanc Health. 2017;5(3):115-121. doi:10.1016/j.peh.2017.03.001

22. Filho E, Aubertin P, Petiot B. The making of expert performers at Cirque du Soleil and the National Circus School: A performance enhancement outlook. J Sport Psychol Action. 2016;7(2):68-79. doi:10.1080/21 520704.2016.1138266

23. Van Rens F, Filho E. Not just clowning around: Investigating psychological mechanisms underlying accidents in a heterogeneous group of contemporary circus artists. Psychol Aesthet Creat Arts. Published online 2019.

24. Van Rens F, Heritage B. Mental health of circus artists: Psychological resilience, circus factors, and demographics predict depression, anxiety, stress, and flourishing. Psychol Sport Exerc. 2021;53:1-9.

25. Filho E, Pierini D, Robazza C, Tenenbaum G, Bertollo M. Shared mental models and intra-team psychophysiological patterns: A test of the juggling paradigm. J Sports Sci. 2017;35(2):112-123. doi:10.108 $\underline{0 / 02640414.2016 .1158413}$

26. Menard J, Halle M. Circus also needs performance psychology: Facts and realities of consulting at Cirque du Soleil. In: Becoming a Sport, Exercise, and Performance Psychology Professional: A Global Perspective. Psychology Press; 2014:127-134.

27. Filho E, Rettig J. Team coordination in high-risk circus acrobatics. Interact Stud. 2018;19(3):499-518. d oi:10.1075/is.16035.fil

28. Shrier I, Halle M. Psychological predictors of injuries in circus artists: An exploratory study. $\mathrm{Br} J$ Sports Med. 2011;45(5):433-436. doi:10.1136/bjsm.20 $\underline{09.067751}$

29. Day MC, Thatcher J, Greenlees I, Woods B. The causes of psychological responses to lost move syndrome in national level trampolinists. J Appl Sport Psychol. 2006;18(2):151-166. doi:10.1080/1041320060 0653782

30. Burtt J, Lavers K. Re-imagining the development of circus artists for the twenty-first century. Theatr Danc Perform Train. 2017;8(2):143-155. doi:10.1080/1 9443927.2017.1316305

31. Ekstrand J, Lundqvist D, Davison M, et al. Communication quality between the medical team and the head coach/manager is associated with injury burden and player availability in elite football clubs. Br J Sports Med. 2019;53:304-308.
32. Karol RL. Team models in neurorehabilitation: Structure, function, and culture change. NeuroRehabilitation. 2014;34(4):655-669. doi:10.3233/ NRE-141080

33. Vaughan J, Mallett CJ, Davids K, Potrac P, LópezFelip MA. Developing creativity to enhance human potential in sport: A wicked transdisciplinary challenge. Front Psychol. 2019;10:1-16. doi:10.3389/fp syg.2019.02090

34. Meyer B, Merkur A, Massey W, et al. The realities of working in elite sport. Applied Sport Science and Medicine. 2014;2014;137-142:137-142.

35. Hess CW, Gnacinski SL, Meyer BB. A review of the sport-injury and rehabilitation literature: From abstraction to application. Sport Psychol. 2019;33(3):232-243. doi:10.1123/tsp.2018-0043

36. Ekstrand J, Lundqvist D, Lagerbäck L, et al. Is there a correlation between coaches' leadership styles and injuries in elite football teams? A study of 36 elite teams in 17 countries. Br J Sports Med. 2018;52:527-531.

37. Faltus J, Criss C, Grooms D. Shifting focus: A clinician's guide to understanding neuroplasticity for anterior cruciate ligament rehabilitation. Curr Sports Med Rep. 2020;19,76-83:76-83.

38. Grooms D, Appelbaum G, Onate J. Neuroplasticity following anterior cruciate ligament injury: A framework for visual-motor training approaches in rehabilitation. J Orthop Sports Phys Ther. 2015;45:381-393.

39. Ungar M, Ghazinour M, Richter I. Annual research review: What is resilience within the social ecology of human development? J Child Psychol Psychiatry. 2013;54:348-366.

40. Stuckey M, Richard V, Decker A, Aubertin P, Kriellaars D. Supporting holistic wellbeing for performing artists during the COVID-19 pandemic and recovery. Front Psychol. 2021;12:1-8. doi:10.3389/ fpsyg.2021.577882

41. Jefferies P. Physical literacy and resilience: The role of positive challenges. Sci Bonheur. 2020;5:11-26.

42. Van Raalte JL, Brewer BW, Cornelius AE, Keeler M, Gudjenov C. Effects of a mental warmup on the workout readiness and stress of college student exercisers. J Funct Morphol Kinesiol. 2019;4(3):42. do i:10.3390/ifmk4030042 
43. Gastin PB, Meyer D, Robinson D. Perceptions of wellness to monitor adaptive responses to training and competition in elite Australian football. J Strength Cond Res. 2013;27(9):2518-2526. doi:10.1519/jsc.0b01 $\underline{3 e 31827 f d 600}$
44. Govus AD, Coutts A, Duffield R, Murray A, Fullagar H. Relationship between pretraining subjective wellness measures, player load, and ratingof-perceived-exertion training load in American college football. Int J Sports Physiol Perform. 2018;13(1):95-101. doi:10.1123/ijspp.2016-0714

45. Decker A, Aubertin P, Kriellaars D. Sleep and fatigue of elite circus student-artists during one year of training. Med Prob Perform Art. 2019;34:125-131. 